\title{
Possible solar origin of the 1,470-year glacial climate cycle demonstrated in a coupled model
}

\author{
Holger Braun ${ }^{1}$, Marcus Christl ${ }^{1}$, Stefan Rahmstorf ${ }^{2}$, Andrey Ganopolski ${ }^{2}$, Augusto Mangini ${ }^{1}$, Claudia Kubatzki ${ }^{3}$, \\ Kurt Roth ${ }^{4} \&$ Bernd Kromer ${ }^{1}$
}

Many palaeoclimate records from the North Atlantic region show a pattern of rapid climate oscillations, the so-called DansgaardOeschger events, with a quasi-periodicity of $\sim 1,470$ years for the late glacial period ${ }^{1-6}$. Various hypotheses have been suggested to explain these rapid temperature shifts, including internal oscillations in the climate system and external forcing, possibly from the Sun ${ }^{7}$. But whereas pronounced solar cycles of $\sim 87$ and $\sim 210$ years are well known $^{8-12}$, a $\sim 1,470$-year solar cycle has not been detected ${ }^{8}$. Here we show that an intermediate-complexity climate model with glacial climate conditions simulates rapid climate shifts similar to the Dansgaard-Oeschger events with a spacing of 1,470 years when forced by periodic freshwater input into the North Atlantic Ocean in cycles of $\sim 87$ and $\sim 210$ years. We attribute the robust 1,470-year response time to the superposition of the two shorter cycles, together with strongly nonlinear dynamics and the long characteristic timescale of the thermohaline circulation. For Holocene conditions, similar events do not occur. We conclude that the glacial 1,470-year climate cycles could have been triggered by solar forcing despite the absence of a 1,470-year solar cycle.

The onset of successive Dansgaard-Oeschger (DO) events, as documented in Greenland ice-cores ${ }^{1,2}$ for example, is typically spaced by $\sim 1,470$ years or integer multiples thereof ${ }^{13,14}$. Because deviations from this cyclicity are small, often less than 100-200 years ${ }^{15}$, external forcing (solar or orbital) was suggested to trigger DO events ${ }^{6,15,16}$. However, neither orbital nor solar forcing shows a 1,470-year frequency. Spectral analysis performed on records of cosmogenic nuclides ${ }^{8-11}$, which are commonly used as proxies for solar variability $^{12}$, indicates the possible existence of pronounced and stable $\mathrm{e}^{10,11}$ centennial-scale solar cycles (the DeVries-Suess and Gleissberg cycles with periods near 210 and 87 years $^{10,11}$ ) but does not reveal a 1,470-year cycle ${ }^{8}$. However, the DeVries and Gleissberg cycles are close to prime factors of 1,470 years $(1,470 / 7=210$; $1,470 / 17 \approx 86.5$ ). The superposition of two such frequencies could result in variability that repeats with a 1,470 -year period.

Here we propose that these two solar frequencies could have synchronized the glacial 1,470-year climate cycle. Support for the idea that a multi-century climate cycle might be linked with centuryscale solar variability comes from Holocene data: a multi-centennial drift-ice cycle in the North Atlantic was reported ${ }^{17}$ to coincide with "rapid (100- to 200-year), conspicuously large-amplitude variations" in the production rates of the cosmogenic isotopes ${ }^{14} \mathrm{C}$ and ${ }^{10} \mathrm{Be}$. To test our hypothesis, we force the coupled climate system model CLIMBER-2 (version 3) with the two solar frequencies. Earlier simulations with this model showed that, when forced by periodic and/or stochastic variations in the freshwater flux into the northern Atlantic, abrupt glacial warming events are triggered that reproduce many features of the observed DO events, including the characteristic time evolution and spatial pattern of these events and also the phase relation of the Antarctic response ${ }^{18-20}$. In the model, the events represent rapid transitions between a stadial ('cold') and an interstadial ('warm') mode of the North Atlantic thermohaline circulation, triggered by a threshold process.

Following these simulations, we here force the model with variations in freshwater input consisting of two sinusoidal components with periods $T_{1}=1,470 / 7(=210)$ years and $T_{2}=1,470 / 17$ $(\approx 86.5)$ years, representing the DeVries and Gleissberg solar cycles. Our focus is the frequency in the glacial climate response (which needs to be studied with a model allowing sufficiently long

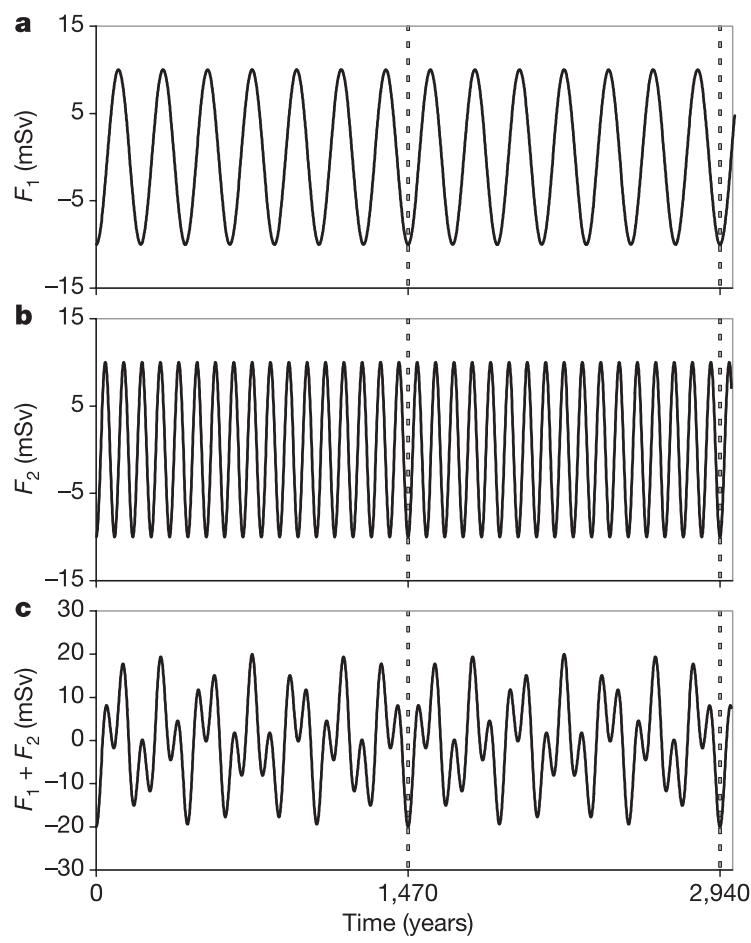

Figure 1 | Applied freshwater forcing in the simulation. a, b, To represent the DeVries and Gleissberg solar cycles we consider two sinusoidal components: DeVries component $F_{1}$ with period $T_{1}=210$ years (a) and Gleissberg component $F_{2}$ with period $T_{2} \approx 86.5$ years (b). c, Total forcing $\left(F_{1}+F_{2}\right)$. The dashed lines indicate the period of 1,470 years in the forcing. In the figure, the amplitudes $A_{1}$ and $A_{2}$ are chosen to be $10 \mathrm{mSv}$ and the phases $\varphi_{1}$ and $\varphi_{2}$ to be 0 . The additional offset $K$ is not shown here.

${ }^{1}$ Heidelberg Academy of Sciences, c/o Institute of Environmental Physics, University of Heidelberg, Im Neuenheimer Feld 229,69120 Heidelberg, Germany. ${ }^{2}$ Potsdam Institute for Climate Impact Research, PO Box 601203, 14412 Potsdam, Germany. ${ }^{3}$ Alfred Wegener Institute for Polar and Marine Research, Bussestrasse 24,27570 Bremerhaven, Germany. ${ }^{4}$ Institute of Environmental Physics, University of Heidelberg, Im Neuenheimer Feld 229, 69120 Heidelberg, Germany. 

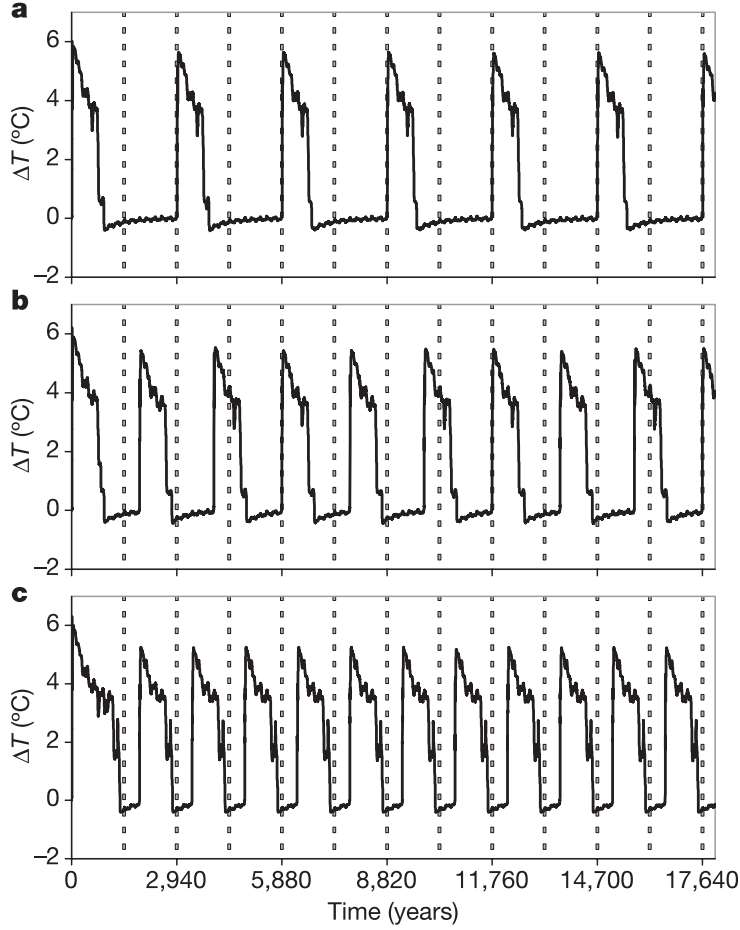

Figure 2 Simulated changes $\Delta T$ in Greenland surface air temperature. The amplitudes $A_{1}$ and $A_{2}$ of the two forcing cycles are chosen to be $10 \mathrm{mSv}$ with different values for the offset $K$. a, $K=-9 \mathrm{mSv}$; b, $K=-14 \mathrm{mSv}$; c, $K=-19 \mathrm{mSv}$. The dashed lines indicate the first-order minima in the forcing (that is, the maxima in the salinity flux), which show a period of 1,470 years (see Fig. 1).

simulations, and correspondingly less resolution and detail). Our study is not aimed at suggesting a certain mechanism for solar influence on freshwater fluxes; this should be studied with more detailed and higher-resolution models. A possible mechanism that has recently been proposed is that solar irradiance variations over the solar periods could generate cyclic salinity anomalies in the North Atlantic by their effect on the thermohaline circulation (THC) ${ }^{21}$. Our simplified approach implies that we assume the known solar frequencies to be present in the hydrological cycle, as suggested previously ${ }^{22,23}$. A more direct implementation of solar forcing in the model (such as one based on records of cosmogenic ${ }^{10} \mathrm{Be}$ ) is hindered by several difficulties: for example, a reliable reconstruction of solar activity over tens of thousands of years would be required, with a resolution and dating precision necessary to show the $\sim 87$-year Gleissberg cycle realistically. This is so far unachievable during the Last Glacial. Furthermore, when transforming solar forcing into variations of freshwater fluxes, a chain of complex and uncertain processes is involved (for example, atmospheric chemistry $^{24-26}$ and the dynamics of continental ice sheets ${ }^{27}$ and sea ice $^{28}$ ) that cannot explicitly be resolved in climate models designed for millennial-scale simulations.

In our simulations, we consider the Last Glacial Maximum (LGM) as the underlying climate state. The total freshwater forcing $F$ (Fig. 1) reads as follows:

$$
F(t)=-A_{1} \cos \left(\omega_{1} t+\varphi_{1}\right)-A_{2} \cos \left(\omega_{2} t+\varphi_{2}\right)+K
$$

with amplitudes $A_{1}$ and $A_{2}$, frequencies $\omega_{1}=2 \pi / T_{1}$ and $\omega_{2}=2 \pi / T_{2}$, and phases $\varphi_{1}$ and $\varphi_{2}$. The constant offset $K$ represents changes in the background climate compared with the LGM. Following earlier simulations ${ }^{18}$, the freshwater perturbation is added to the North Atlantic in the latitude belt $50-70^{\circ} \mathrm{N}$. This area is particularly sensitive to solar forcing: On one hand, variations in the atmospheric heat fluxes can trigger shifts of the sea-ice margin that-because of the insulating effect of sea-ice ${ }^{28}$-result in evaporation anomalies. On the other hand, solar forcing can cause meltwater anomalies by its effect on the mass balance of the ice sheets surrounding this region. The applied amplitudes $A_{1}$ and $A_{2}$ are very small: in the range of $10 \mathrm{mSv}\left(1 \mathrm{~Sv}=1\right.$ Sverdrup $\left.=10^{6} \mathrm{~m}^{3} \mathrm{~s}^{-1}\right)$; that is, about $5 \mathrm{~cm} \mathrm{yr}^{-1}$ in the surface freshwater flux into that area. Although the total forcing does not explicitly have a spectral component of 1,470 years, it repeats with this period because of the combined effect of the applied cycles.

In the response of the model, three different regimes exist: a 'cold regime' in which the THC persists in the stadial mode, a 'warm regime' in which the interstadial mode is stable, and a 'DansgaardOeschger regime' in which cyclic transitions between both modes are excited. These transitions result in abrupt warm events in the model box containing Greenland, similar to DO events (Fig. 2). Figure 3 illustrates the model response for various amplitudes $A$ $\left(A=A_{1}=A_{2}\right)$ and offsets $K$. Events with a spacing of 1,470 years are found within a continuous forcing-parameter range. This 1,470-year timescale in the model response is robust when the phases, the

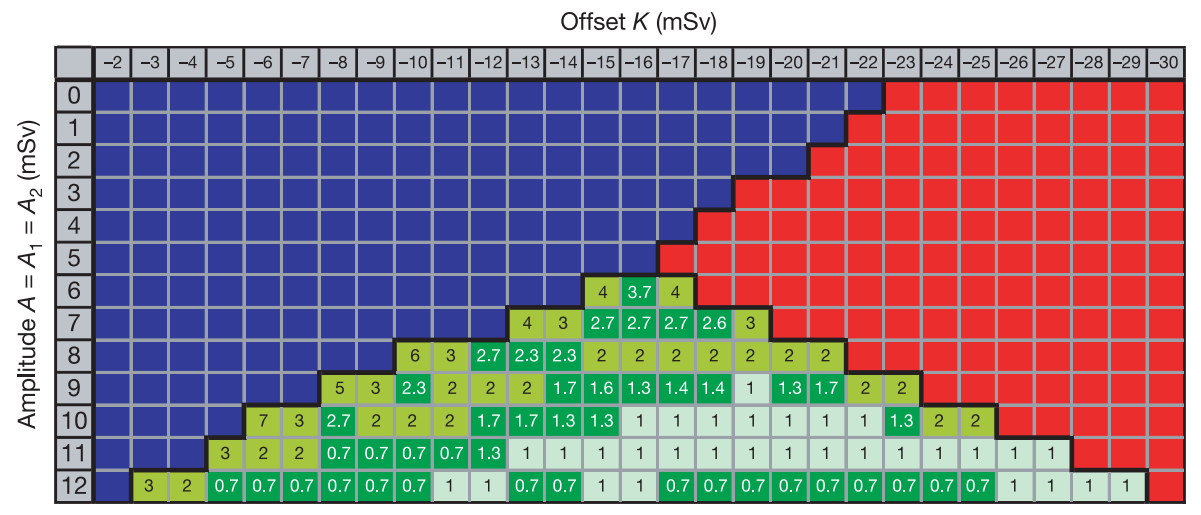

Stadial mode is stable

Interstadial mode is stable
Spacing of 1,470 years

Spacing of integer multiples of 1,470 years

Spacing different from integer multiples of 1,470 years
Figure 3 | Schematic response of the model for different amplitudes $A$ (with $A=A_{1}=A_{2}$ ) and offsets $K$ in the forcing. Upper left area (blue): 'cold' regime (stadial mode is stable). Upper right area (red): 'warm' regime (interstadial mode is stable). Lower area (green): 'Dansgaard-Oeschger' regime (none of the modes is stable). The numbers in this regime indicate the most frequently occurring temporal spacing between successive events (in multiples of 1,470 years) for each combination of $A$ and $K$. 
amplitudes and the periods of the two forcing cycles are changed over some range (Supplementary Information). White noise, when added to the periodic freshwater forcing, acts as an amplifier but does not affect the robustness of the 1,470-year model timescale (Supplementary Information). In the presence of noise, different DO events look different, unlike the identical events occurring for completely regular forcing (Fig. 2). When instead of two sinusoidal cycles a more realistic forcing is applied with spectral properties close to those observed in solar proxies ${ }^{8,10,29}$, a regular 1,470 -year model response can still be obtained (Fig. 4 and Supplementary Information).

The stability of the simulated 1,470-year climate cycle is a plausible consequence of two well-known properties of the THC: its long characteristic timescale, and the high degree of nonlinearity (that is, the threshold character) inherent in the transitions between the two modes of the THC. A very simple conceptual model that only incorporates these two properties is able to mimic key features of CLIMBER-2, for example the existence of three different regimes in the model response, the frequency conversion between forcing and response (that is, the excitation of millennial-scale spectral components in the model response that do not exist in the forcing) and the amplitude dependence of the period in the model response. The general idea of this model is that DO events represent highly nonlinear switches between two different climate states corresponding to the stadial and interstadial modes of the glacial THC. A detailed description of the conceptual model, as well as a discussion of its
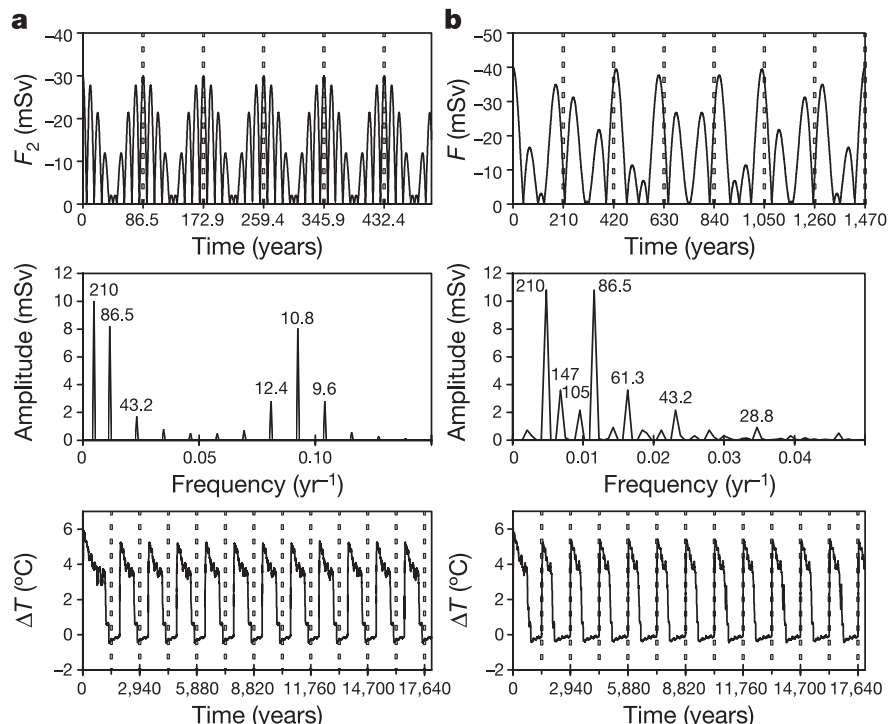

Figure 4 | Model response for non-sinusoidal forcing profiles. a, Roughly 86.5-year Gleissberg cycle implemented as amplitude modulation of a 11-year cycle (representing the Schwabe solar cycle). Top panel: combined Gleissberg/Schwabe forcing component $F_{2}=-A_{2}\left|\cos \left(\omega_{2} t / 2\right) \| \cos \left(\omega_{3} t / 2\right)\right|$. The chosen forcing parameters are $A_{2}=30 \mathrm{mSv}, \omega_{2}=2 \pi / T_{2}\left(T_{2}=1,470 /\right.$ 17 years $\approx 86.5$ years $), \omega_{3}=2 \pi / T_{3}\left(T_{3}=1,470 / 136\right.$ years $\approx 10.8$ years $)$. $|\cos (\omega t / 2)|$ is used because $|\cos (\pi t / T)|$ has a period of $T$. The 210 -year DeVries component $F_{1}$ is considered as an additional cosine cycle, identical to that in Fig. 1a: $F_{1}=-A_{1} \cos \left(\omega_{1} t\right)$ with $A_{1}=10 \mathrm{mSv}$ and $\omega_{1}=2 \pi / T_{1}$ $\left(T_{1}=1,470 / 7\right.$ years $=210$ years $)$. Note: here, the freshwater axis is inverted compared with that in Fig. 1. The total forcing is the sum of both components plus an offset $K=-5 \mathrm{mSv}$. Middle panel: Fourier spectrum of $F_{1}+F_{2}$. The spectrum is discrete because the forcing is periodic. Numbers above peaks are years. Bottom panel: model response. b, Gleissberg cycle modulated by the DeVries cycle. Top panel: combined DeVries/Gleissberg forcing component $F=-A_{1}\left|\cos \left(\omega_{1} t / 2\right) \| \cos \left(\omega_{2} t / 2\right)\right|$. The chosen forcing parameters are $A_{1}=40 \mathrm{mSv}, \omega_{1}=2 \pi / T_{1}\left(T_{1}=1,470 / 7\right.$ years $=210$ years), $\omega_{2}=2 \pi / T_{2}\left(T_{2}=1,470 / 17\right.$ years $\approx 86.5$ years $)$. The total forcing is given by $F$ plus an offset $K=5 \mathrm{mSv}$. Middle panel: Fourier spectrum of $F$. Bottom panel: model response. response to the applied forcing, is given in the Supplementary Information.

A key aspect of our concept is that the events are triggered by very sharp peaks in the forcing, with duration of only decades. This can explain the puzzling regularity in the timing of DO events in the Greenland data, which were reported to deviate by no more than $\sim 10 \%$ from exact multiples of 1,470 years $^{15}$. If the events were triggered by broad peaks (for example by some sinusoidal-like forcing cycle of a 1,470-year period), much larger deviations from exact timing would be expected in the 'noisy' climate system. Hence, the exact timing of the more recent DO events (ages between 10,000 and 50,000 years) is a strong observational indication that they are synchronized by shorter cycles.

We have demonstrated that a coupled ocean-atmosphere climate model can reproduce DO events with a robust spacing of 1,470 years when forced by the superposition of two freshwater cycles with much shorter periods near 87 and 210 years. A frequency of 1,470 years is therefore not found in the forcing; it is found only in the model response. We illustrated that this frequency conversion between forcing and response is a plausible consequence of highly nonlinear dynamics inherent in the simulated DO events. Our results indicate that the observed 1,470-year climate cycle could have originated from solar variability despite the lack of a 1,470-year spectral contribution in records of solar activity. Moreover, the 1,470-year climate response in the simulation is restricted to glacial climate and cannot be excited for substantially different (such as Holocene) boundary conditions; for these, the model response shows the frequencies of the applied forcing ( $\sim 86.5$ and 210 years), as also documented in various climate archives $^{22,23,30}$. Thus, our mechanism for the glacial $\sim 1,470$-year climate cycle is also consistent with the lack of a clear and pronounced 1,470-year cycle in Holocene climate archives.

\section{Received 26 May; accepted 10 August 2005.}

1. Bond, G. et al. Correlations between climate records from North Atlantic sediments and Greenland ice. Nature 365, 143-147 (1993).

2. Grootes, P. M., Stuiver, M., White, J. W. C., Johnson, S. \& Jouzel, J. Comparison of the oxygen isotope records from the GISP2 and GRIP Greenland ice cores. Nature 366, 552-554 (1993)

3. Sachs, J. P. \& Lehman, S. J. Subtropical North Atlantic temperatures 60,000 to 30,000 years ago. Science 286, 756-759 (1999).

4. Spötl, C. \& Mangini, A. Stalagmite from the Austrian Alps reveals DansgaardOeschger events during isotope stage 3: Implications for the absolute chronology of Greenland ice cores. Earth Planet. Sci. Lett. 203, 507-518 (2002).

5. Voelker, A. H. L. et al. Global distribution of centennial-scale records for marine isotope stage (MIS) 3: a database. Quat. Sci. Rev. 21, 1185-1212 (2002).

6. Schulz, M. On the 1470-year pacing of Dansgaard-Oeschger warm events. Paleoceanography 17, doi:10.1029/2000PA000571 (2002).

7. van Geel, B. et al. The role of solar forcing upon climate change. Quat. Sci. Rev. 18, 331-338 (1999)

8. Stuiver, M. \& Braziunas, T. F. Sun, ocean, climate and atmospheric ${ }^{14} \mathrm{CO}_{2}$ : an evaluation of causal and spectral relationships. Holocene 3, 289-305 (1993).

9. Beer, J. et al. in The Sun as a Variable Star: Solar and Stellar Irradiance Variations (eds Pap, J. M., Fröhlich, H., Hudson, S. \& Solanki, S. K.) 291-300 (Cambridge Univ. Press, Cambridge, 1994).

10. Peristykh, A. N. \& Damon, P. E. Persistence of the Gleissberg 88-year solar cycle over the last $\sim 12,000$ years: Evidence from cosmogenic isotopes. J. Geophys. Res. 108, 1003, doi:10.1029/2002JA009390 (2003).

11. Wagner, G. et al. Presence of the solar de Vries cycle ( $\sim 205$ years) during the last ice-age. Geophys. Res. Lett. 28, 303-306 (2001).

12. Lean, J. \& Rind, D. Climate forcing by changing solar radiation. J. Clim. 11, 3069-3094 (1998).

13. Alley, R. B., Anandakrishnan, S. \& Jung, P. Stochastic resonance in the North Atlantic. Paleoceanography 16, 190-198 (2001)

14. Alley, R. B., Anandakrishnan, S., Jung, P. \& Clough, A. in The Oceans and Rapid Climate Change: Past, Present and Future (eds Seidov, D., Maslin, M. \& Haupt, B. J.) 57-68 (AGU Geophys. Monogr. 126, Washington, D.C., 2001).

15. Rahmstorf, S. Timing of abrupt climate change: A precise clock. Geophys. Res. Lett. 30, 1510, doi:10.1029/2003GL017115 (2003).

16. Keeling, C. D. \& Whorf, T. P. The 1,800-year oceanic tidal cycle: A possible cause of rapid climate change. Proc. Natl Acad. Sci. USA 97, 3814-3819 (2000)

17. Bond, G. et al. Persistent solar influence on North Atlantic climate during the Holocene. Science 294, 2130-2136 (2001). 
18. Ganopolski, A. \& Rahmstorf, S. Rapid changes of glacial climate simulated in a coupled climate model. Nature 409, 153-158 (2001).

19. Ganopolski, A. \& Rahmstorf, S. in The Oceans and Rapid Climate Change: Past, Present and Future (eds Seidov, D., Maslin, M. \& Haupt, B. J.) 261-275 (AGU Geophys. Monogr. 126, Washington, D.C., 2001).

20. Ganopolski, A. \& Rahmstorf, S. Abrupt glacial climate changes due to stochastic resonance. Phys. Rev. Lett. 88, 038501 (2002).

21. Weber, S. L., Crowley, T. J. \& van der Schrier, G. Solar irradiance forcing of centennial climate variability during the Holocene. Clim. Dyn. 22, 539-553 (2004)

22. Neff, U. et al. Strong coherence between solar variability and the monsoon in Oman between 9 and 6 kyr ago. Nature 411, 290-293 (2001).

23. Hodell, D. A., Brenner, M., Curtis, J. H. \& Guilderson, T. Solar forcing of drought frequency in the Maya lowlands. Science 292, 1367-1370 (2001).

24. Haigh, J. D. The role of stratospheric ozone in modulating the solar radiative forcing of climate. Nature 370, 544-546 (1994)

25. Shindell, D., Rind, D., Balachandran, N., Lean, J. \& Lonergan, P. Solar cycle variability, ozone, and climate. Science 284, 305-308 (1999).

26. Carslaw, K. S., Harrison, R. G. \& Kirkby, J. Cosmic rays, clouds and climate. Science 298, 1732-1737 (2002).

27. Schmittner, A., Yoshimori, M. \& Weaver, A. J. Instability of glacial climate in a model of the ocean-atmosphere-cryosphere system. Science 295, 1489-1493 (2002).
28. Lohmann, G. \& Gerdes, R. Sea ice effects on the sensitivity of the thermohaline circulation in simplified atmosphere-ocean-sea ice models. J. Clim. 11, 2789-2803 (1998).

29. Ogurtsov, M. G., Nagovitsyn, Y. A., Kocharov, G. E. \& Jungner, H. Long-period cycles of the Sun's activity recorded in direct solar data and proxies. Solar Phys. 211, 371-394 (2002)

30. Ram, M. \& Stolz, M. Possible solar influences on the dust profile of the GISP2 ice core from Central Greenland. Geophys. Res. Lett. 26, 1043-1046 (1999).

Supplementary Information is linked to the online version of the paper at www.nature.com/nature.

Acknowledgements We thank N. Latuske for insight into spectral analysis M. Claussen, R. Calov and E. Bauer for support and helpful comments; and the late G. Bond for his support and for many discussions that helped to improve the quality of this study. C.K. was funded by a subcontract to a project of the Bundesministerium für Bildung und Forschung (BMBF).

Author Information Reprints and permissions information is available at npg.nature.com/reprintsandpermissions. The authors declare no competing financial interests. Correspondence and requests for materials should be addressed to H.B. (holger.braun@iup.uni-heidelberg.de). 